\title{
Proposta de utilização de metodologia didática alternativa para o estudo da anatomia veterinária
}

\author{
Proposal of using alternative teaching methods \\ for the veterinary anatomy study
}

\begin{abstract}
Gabriela Santos Freitas, Thais Cristina Belondre Andrade, Laís Stephanie Barbosa Baptista, Dala Kezen Vieira Hardman Leite, Rômulo Ferreira de Assumção
\end{abstract}

Universidade Iguaçu (UNIG), Nova Iguaçu, RJ, Brasil

\section{Resumo}

O objetivo desse trabalho foi propor uma metodologia para facilitar o aprendizado e diminuir o uso de cadáveres nos cursos de medicina veterinária por meio de métodos alternativos para desenvolvimento de modelos anatômicos. Para isso, foram utilizados diversos materiais como massa de biscuit, glicerina, tinta acrílica e cartolina. Empregou-se a técnica de glicerinação para conservar uma peça com estômago, baço, duodeno, pâncreas e omento, e outra com bexiga e próstata. Esta técnica é mais duradoura e não causa riscos à saúde, como ocorre com o uso do formol. Foi confeccionado, também, um modelo de bexiga com massa de biscuit, e tintas foram utilizadas para pintar os ossos do crânio de um cão. Em cartolinas foram desenhados esquemas do pulmão, coração e sistema urinário, o que facilita a visualização de estruturas difíceis de serem observadas através da dissecção. Todos esses modelos foram apresentados a 40 discentes que responderam a uma pesquisa de satisfação avaliando os novos métodos, com $100 \%$ de aceitação. Desta forma, conclui-se que a utilização de recursos didáticos alternativos contribui de maneira positiva no processo ensino-aprendizagem e nas questões morais envolvidas.

Palavras-chave: Modelos anatômicos. Morfologia. Modelos didáticos. Metodologia ativa.

\begin{abstract}
The objective of this work was to propose a methodology to facilitate learning and to decrease the use of cadavers in veterinary medicine courses through alternative methods for the development of anatomical models. For
\end{abstract}


this, several materials were used, such as biscuit dough, glycerin, acrylic paint and cardboard. Glycerination technique was used to conserve a piece with stomach, spleen, duodenum, pancreas and omentum, and another with bladder and prostate. This technique is more durable and does not cause health risks, like the formaldehyde use does. A bladder model was also made with biscuit dough. The paints were used to paint the bones of a dog's skull. On the cards, schemes of the lung, heart and urinary system were designed, which facilitates the visualization of structures difficult to be observed through dissection. All of these models were presented to 40 students who answered a satisfaction survey evaluating the new methods, with $100 \%$ acceptance of these. Thus, we conclud that the use of these didactic resources contributes positively in the teaching-learning process and for the moral issues involved.

Keywords: Anatomical models. Morphology. Didactic models. Active methodology.

\section{Introdução}

O uso de animais em pesquisas é uma prática antiga. Desde 200 a.C. há registros de dissecção de animais por Alcméon (500 a.C.), Aristóteles (384-322 a.C.) e Herófilo (335-280 a.C.), e experimentos com animais mortos ou vivos por Erasístrato (310-250 a.C.) e Hipocrátes (460-370 a.C.) (Lima e Freitas, 2009; Guimarães et al., 2016; Silva et al., 2016). Galeno (130-200 d.C.) praticou vivissecção em mais de oitenta espécies animais para verificar o comportamento dos sistemas (Baeder et al., 2012).

No tempo de Descartes (1596-1650), uma visão difundida, que ele mesmo tinha, era que os animais viviam simplesmente como máquinas, sem demonstrar emoções, motivo este que justificava a sua experimentação (Tinoco e Correia, 2010). No século XVIII, Voltaire foi contrário a essa concepção, afirmando que os animais têm sentidos, sensações, ideias e memórias. Muitos outros pensadores compartilhavam da mesma opinião e, dessa forma, medidas em prol dos animais foram gradativamente tomando conta da experimentação animal (Barros, 2018).
Em 1959, Willian Russel e Rex Burch propuseram o conceito dos três Rs (replacement, reduction, refinement; substituição, redução, refinamento), que se tornou um foco tanto para o bem-estar animal quanto para as comunidades científicas em todo o mundo (Russel e Burch, 1959). A adesão aos três Rs é um dos princípios que orientam as comissões de bioética (Carniatto, 2017), sendo base para a redução do número de animais utilizados, para o aprimoramento das técnicas que os utilizam e para sua substituição (Dipasquale e Hayes, 2001).

A Declaração Universal dos Direitos dos Animais, criada em 1978 pela UNESCO e ainda vigente, prevê no artigo $8^{\circ}$ que a experimentação envolvendo sofrimento físico é incompatível com o direito animal e que técnicas de substituição devem ser desenvolvidas e utilizadas (UNESCO, 1978). Ainda hoje, entretanto, os animais são vistos pela comunidade acadêmica e científica como ferramenta de trabalho (Magalhães e Ortêncio Filho, 2006). A sua utilização no processo de ensinoaprendizagem tem trazido questionamentos e debates públicos há mais de um século (Tréz, 2015).

Muitas universidades têm sido questionadas por grupos de proteção animal quanto à utilização de animais como recursos didáticos, a fim de que essas atividades sejam restringidas ou até mesmo abolidas (Souza et al., 2012). Lima e Freitas (2009) afirmam que o uso de animais é, em muitos casos, somente um recurso utilizado sem o objetivo de possibilitar novas aprendizagens, sendo apenas uma forma de ilustrar determinados conteúdos bem como confirmar habilidades técnicas e conceituais. Além disso, métodos de abordagem alternativos ao estudo e experimentação animais são escassos, fazendo com que, para os alunos, as práticas convencionais pareçam sempre normais e adequadas (Sousa et al., 2015).

Estratégias como o uso de modelos didáticos artesanais para estudo das estruturas anatômicas e técnicas de preservação mais eficientes e duradouras para a diminuição do uso de cadáveres têm sido relatadas por alguns autores como propostas alternativas de aprendizagem (Karam et al., 2016; Farias et al., 2018;). Tais métodos substitutivos surgem em resposta a uma demanda crescente nas universidades, com o objetivo de melhorar a qualidade da educação e, 
principalmente, assegurar que animais não sejam utilizados de forma indiscriminada, levando em conta o valor dos mesmos (Magalhães e Ortêncio Filho, 2006; Seixas et al., 2010; Silva et al., 2014; Tréz, 2015). Para atender a essa demanda existe uma grande variedade de alternativas que podem ser utilizadas, tais quais: modelos, manequins e simuladores mecânicos; filmes e vídeos interativos; simulações computacionais e de realidade virtual; estudos de campo e observacionais (Magalhães e Ortêncio Filho, 2006; Seixas et al., 2010). A utilização dessas técnicas também pode ajudar na visualização de peças pequenas e/ou de difícil acesso na dissecação (Souza et al., 2012; Silva et al., 2014; Karam et al., 2016;).

Dentre as vantagens do emprego de métodos alternativos, podemos citar: menor custo a médio e longo prazo se comparado com a manutenção, manipulação e preparação de animais, além de maior durabilidade; aprendizagem equivalente e muitas vezes superior ao uso tradicional de animais, já que os estudantes aprendem em seu próprio ritmo, podendo repetir o estudo quantas vezes forem necessárias; são condizentes com os princípios éticos, morais ou religiosos de estudantes que se opõem ao uso de animais para estas finalidades; e conforme a necessidade ou o conteúdo a ser ensinado, as alternativas podem ser combinadas, complementando-se umas às outras (Magalhães e Ortêncio Filho, 2006; Seixas et al., 2010; Karam et al., 2016; Silva et al, 2016).

Esse trabalho tem como objetivo propor métodos alternativos para o desenvolvimento de modelos anatômicos que sejam didáticos e facilitem o aprendizado, diminuindo o uso de cadáveres.

\section{Material e métodos}

Modelos anatômicos foram confeccionados utilizando métodos e materiais alternativos como massa de biscuit, glicerina, cartolina e tintas acrílica e aquarela. Todas as peças utilizadas foram oriundas de cadáveres doados para o Laboratório Anatômico da Universidade Iguaçu (UNIG), em Nova Iguaçu, RJ, onde também foi realizado todo o trabalho no ano de 2019. O projeto foi aprovado pelo comitê de ética no uso de animais (CEUA) sob o protocolo 004/2018.

Massa de biscuit branca, colorida com corante vermelho, foi utilizada para fazer o molde de uma bexiga e suas estruturas, como ureteres e uretra. Utilizou-se, também, tinta acrílica e verniz para pintar de diferentes cores os ossos e acidentes anatômicos do crânio de um cão.

Esquemas do sistema urogenital, coração, pulmão e suas estruturas foram desenhados em cartolina, em dimensões de $1 \mathrm{~m} \times 0,6 \mathrm{~m}$, utilizandose caneta preta. $O$ sistema urogenital foi pintado com tinta aquarela.

Peças anatômicas com estômago, duodeno, pâncreas, omento e baço (bloco 1) e próstata e bexiga (bloco 2) foram preparadas por meio de glicerinação, processo que consiste em manter os tecidos mais próximos do natural e sem a imersão em soluções conservadoras. Para isso, primeiramente usou-se formol como fixador, em concentração de $10 \%$, durante seis a doze horas. Posteriormente, as peças foram colocadas submersas em glicerina por algumas semanas. Para o bloco 1 foram necessárias duas semanas; para o bloco 2, uma semana. Assim que ficaram prontas, com um aspecto firme e mais claro, utilizou-se papel absorvente para a secagem até as peças estarem secas o suficiente para serem manuseadas sem luvas.

Os métodos propostos foram utilizados durante uma aula de anatomia, ministrada a 40 alunos do segundo período de medicina veterinária da UNIG. Ao final da aula, realizou-se uma pesquisa de satisfação a fim de averiguar a aceitação da metodologia aplicada.

\section{Resultados e discussão}

Os órgãos como estômago, duodeno, pâncreas, omento, baço, próstata e bexiga, enchidos com maravalha, foram glicerinados (Figuras 1 e 2), pois esta técnica confere uma maior proximidade com o órgão in natura e menor toxicidade do que peças anatômicas formolizadas. Como afirmado por Karam et al. (2016), além de muito eficiente para a conservação, esta técnica é didática e não oferece riscos à saúde. 
Foram confeccionados desenhos em forma de cartazes, com dimensão de $1 \mathrm{~m} \times 0,6 \mathrm{~m}$, e esquemas que foram colocados nas paredes do anatômico, como coração (Figura 3), pulmão (Figura 4) e sistema urogenital. Deste modo, preservou-se fielmente a topografia original dos vasos e nervos, o que é difícil na dissecção (Silva et al., 2014). Foi feita, também, uma pintura dos ossos de um crânio

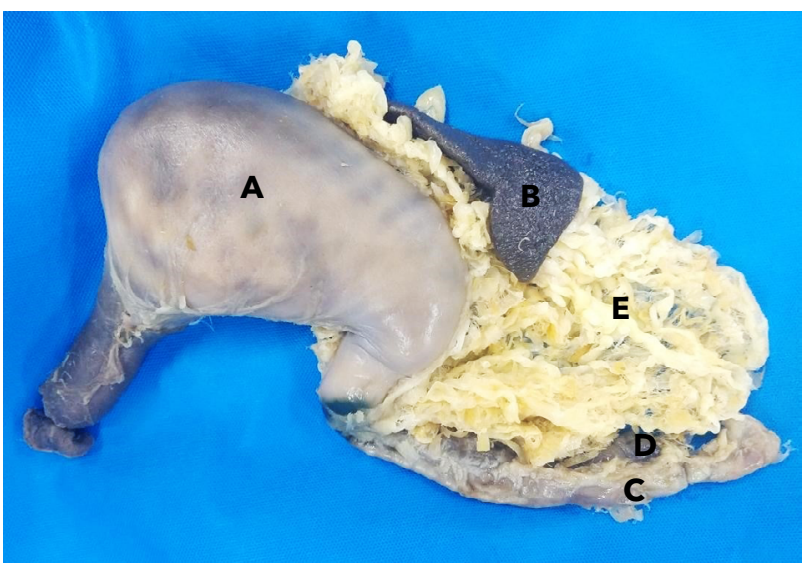

Figura 1 - Figura dos órgãos de cão em glicerina. A estômago; B - baço; C - duodeno; D - pâncreas; E - omento. de cão (Figura 5), onde cada osso foi colorido com uma cor diferente, proporcionando uma maior facilidade de entendimento dos limites ósseos do crânio e a demonstração de vários acidentes ósseos em uma única peça. Trata-se, de acordo com Farias et al. (2018), de um facilitador para o entendimento principalmente da posição anatômica, bem como da relação da região com as demais áreas.

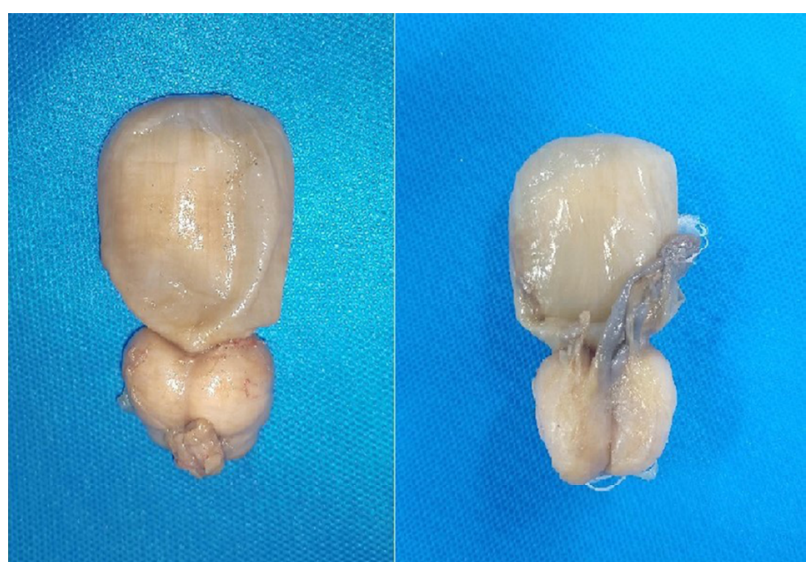

Figura 2 - Peças glicerinadas de bexiga e próstata de cão.
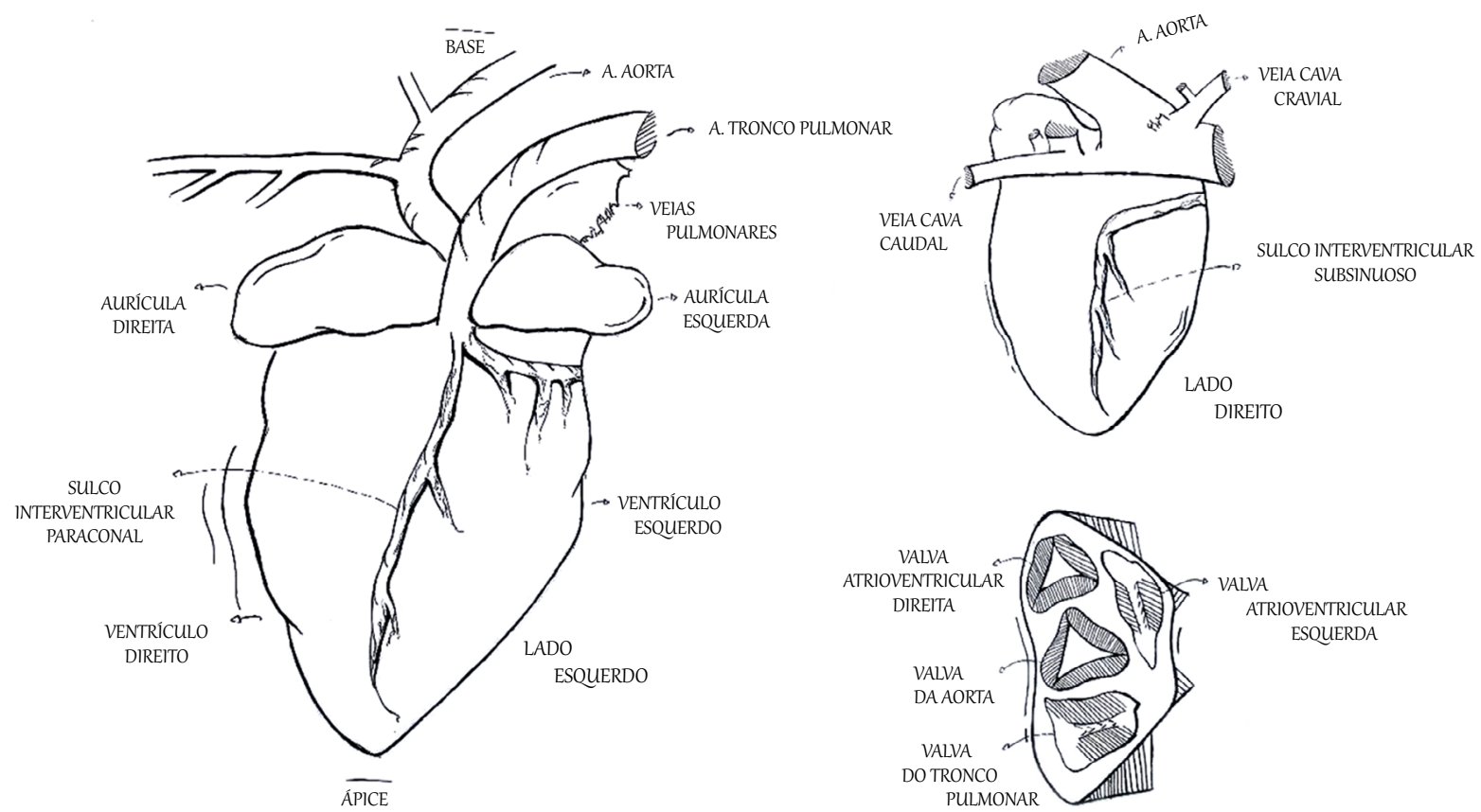

Figura 3 - Esquema de coração de cão desenhado em cartolina.

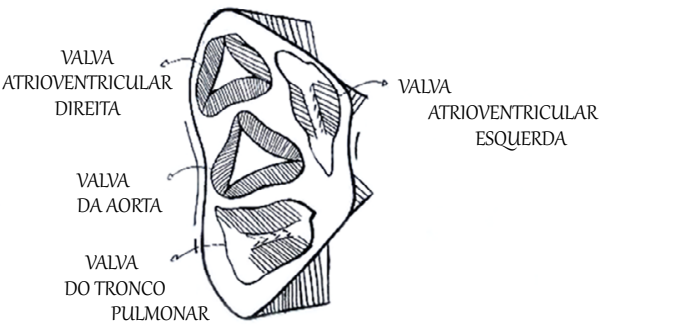




\section{Pulmão (cão)}

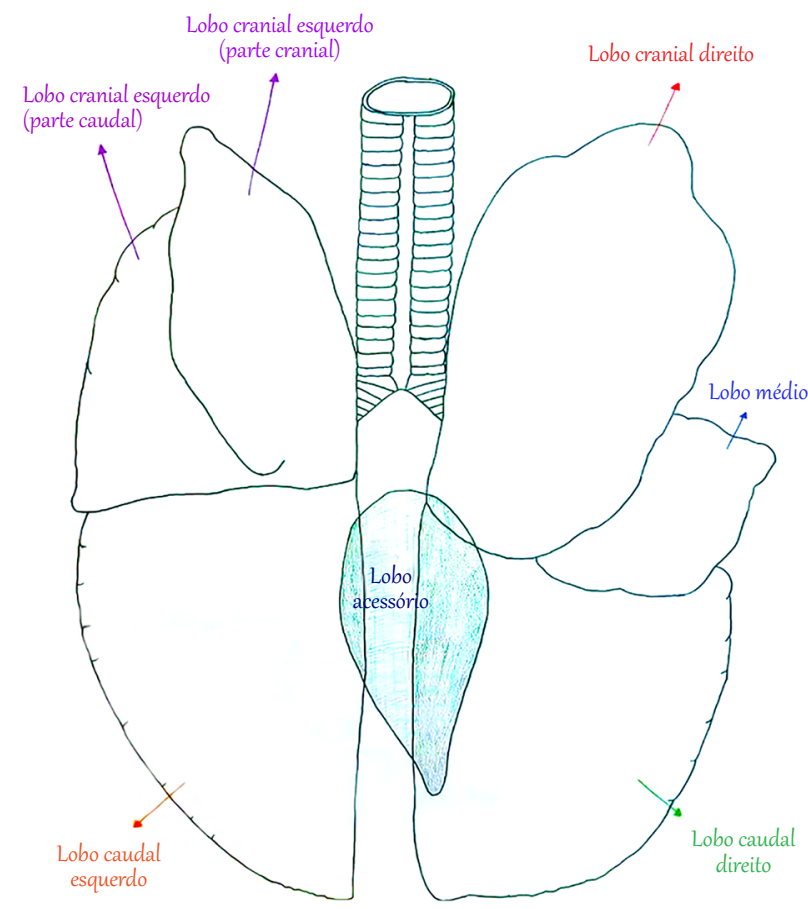

Figura 4 - Esquema de pulmão de cão desenhado em cartolina.

Como realizado por Silva et al.(2014), a massa de biscuit foi utilizada para confeccionar um modelo de bexiga (Figura 6) para melhor visualização das estruturas presentes, como uretra e ureteres. Todos esses métodos são formas artesanais e didáticas para o ensino da anatomia veterinária (Souza et al., 2012). Seixas et al. (2010) afirmam que através desses métodos substitutivos, os estudantes podem repetir experimentos diversas vezes, desenvolvendo habilidades motoras, já que não há restrição de tempo para a prática.

Como forma de avaliação, aplicou-se uma pesquisa de satisfação para 40 discentes do segundo período do curso de medicina veterinária da UNIG. Cem por cento foram a favor dos métodos alternativos e afirmaram que sua utilização nas aulas foi importante para melhor assimilação do conteúdo. A maioria já era a favor dos métodos propostos antes de começarem a estudar anatomia animal, mostrando que esse pensamento já está difundido e que estes alunos refletiram nas questões éticas envolvidas, mesmo não sendo uma prática comum no meio acadêmico. Como afirmaram os autores Barros (2018), Seixas et al. (2010) e Carniatto (2017), a partir da divulgação de ideias que levam em conta o valor do animal, há um aumento de debates e novos conhecimentos acerca do tema no meio científico e universitário.

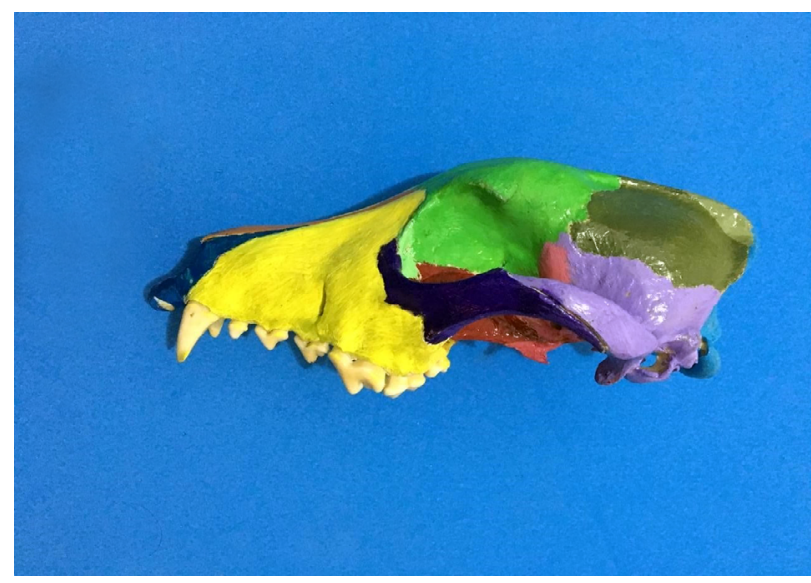

Figura 5 - Crânio de cão colorido com diferentes cores.

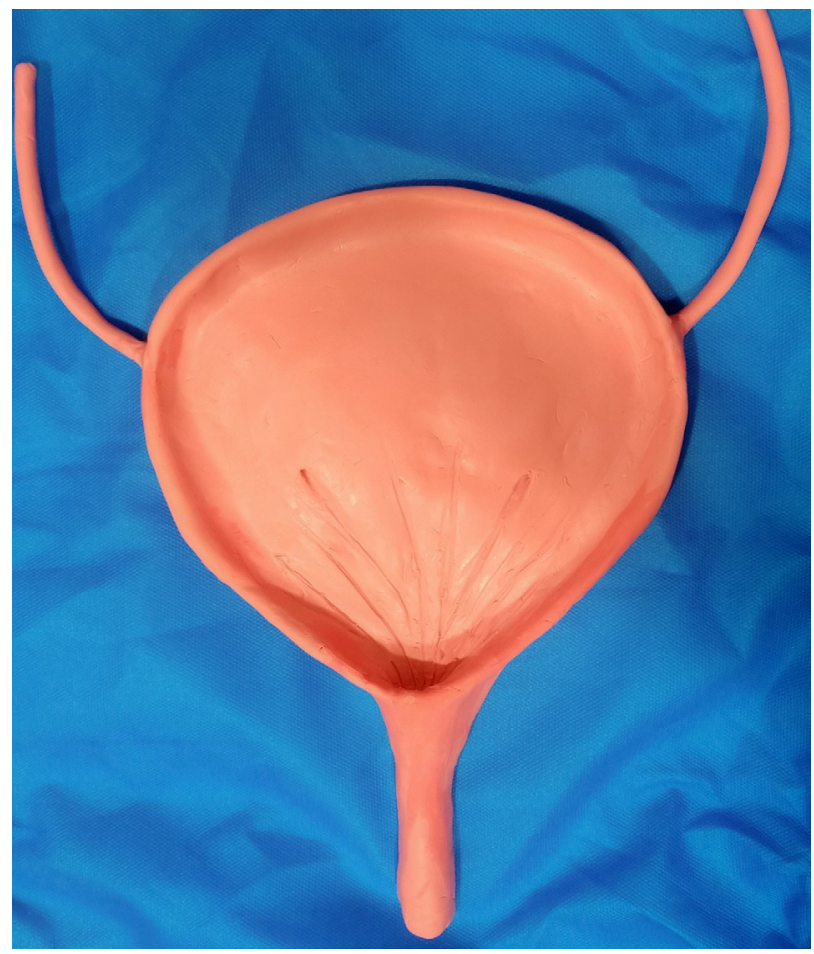

Figura 6 - Modelo de bexiga de cão em biscuit. 


\section{Conclusão}

As metodologias elaboradas durante 0 trabalho se mostraram tão eficazes no processo de ensino-aprendizagem quanto a utilização de cadáveres, muitas vezes mostrando-se até superiores para o entendimento de algumas estruturas. Dessa forma, verificou-se que é possível a diminuição do uso de cadáveres, além de essas metodologias terem um custo menor a longo prazo e uma menor necessidade de manutenção das peças.

\section{Referências}

Barros GA. O direito dos animais e o bem-estar na sua utilização para fins científicos em benefício da saúde humana. FIBRA Lex. 2018;3(3):4-17.

Baeder FM, Padovani MCRL, Moreno DCA, Delfino CS. Percepção histórica da bioética na pesquisa com animais: possibilidades. Bioethikos. 2012;6(3):313-20.

Carniatto $\mathrm{CHO}$. Propostas pedagógicas substitutivas ao uso de animais no ensino superior: Uma revisão. Pubvet. 2017;11(5):443-51.

Dipasquale LC, Hayes AW. Acute toxicity and eye irritancy. In: Hayes AW. Principles and methods of toxicology. 4 ed. Londres: Taylor \& Francis; 2001. p. 853-916.

Farias WA, Machado ACLC, Coutinho BG, Oliveira EL, Lima WJM. Demarcação de regiões do crânio humano através da técnica de pintura: produção de material didático para aula de anatomia humana funcional. Rev Campo Saber. 2018;4(6):51-4.

Guimarães MV, Freire JEC, Menezes LMB. Utilização de animais em pesquisas: breve revisão da legislação no Brasil. Rev Bioet. 2016;24(2):217-24.

Karam RG, Cury FS, Ambrósio CE, Mançanares CAF. Uso da glicerina para a substituição do formaldeído na conservação de peças anatômicas. Pesq Vet Bras. 2016;36(7):671-5.
Lima KC, Freitas GCC. A manipulação de animais é necessária para a aprendizagem de conceitos zoológicos no ensino básico? VII Encontro Nacional de Pesquisadores em Educação em Ciências; 8 nov 2009; Florianópolis, SC.

Magalhães $M$, Ortêncio Filho $H$. Alternativas ao uso de animais como recurso didático. Arq Cienc Vet Zool. 2006;9(2):147-54.

Russel WMS, Burch RL. The principles of humane experimental technique. Springfield: Charles C. Thomas; 1959.

Seixas MM, Virgens JHA, Melo SMB, Van Herk AGS. Consciência na substituição do uso de animais no ensino: aspectos históricos, éticos e de legislação. Rev Bras Direito Anim. 2010;5(6):71-96.

Silva AA, Onety Jr RT, Merini LR, Pereira EN, Brito LS. O uso do biscuit como ferramenta complementar ao ensino de anatomia humana: um relato de extensão universitária. Rev Cienc Ext. 2014;10(2):47-54.

Silva RM, Santori RT, Miranda JC. Experimentação animal e ensino. SaBios Rev Saude e Biol. 2016;11(1):90-100.

Sousa TC, Pontes KCS, Machado JP, Lacerda DG. Elaboração de técnica alternativa para o ensino prático da homeostasia em medicina veterinária. Rev Cient Univiçosa. 2015;7(1):432-8.

Souza ALM, Assumção RF, Guimarães LF, Rodrigues ABF. Utilização de métodos didáticos alternativos para o estudo da anatomia veterinária. Pubvet. 2012;6(27):1423-8.

Tinoco IAP, Correia MLA. Reflexões éticas sobre a vivissecção no Brasil. XIX Encontro Nacional do CONPEDI; 9-12 jun 2010; Fortaleza, CE. Florianópolis: Fundação Boiteux; 2010. p. 6549-77.

Tréz TA. A caracterização do uso de animais no ensino a partir da percepção de estudantes de ciências biológicas e da saúde. Hist Cienc Saude-Manguinhos. 2015;22(3):863-80.

UNESCO. Declaração Universal dos Direitos dos Animais. Bruxelas: UNESCO; 1978. 